\title{
MicroRNA-195 rescues ApoE4-induced cognitive deficits and lysosomal defects in Alzheimer's disease pathogenesis
}

 \\ Farida El Gaamouch ${ }^{2}$ - Gregory Elder ${ }^{1,2}$ - Mary Sano ${ }^{1,6,7}$ - Alison Goate (iD ${ }^{8,9} \cdot$ Julia TCW (D) $^{8,9}$. \\ Vahram Haroutunian $^{6,7,8,10}$ - Bin Zhang ${ }^{3,4}$ - Dongming Cai CD $^{1,2,4,7,9}$
}

Received: 30 October 2019 / Revised: 3 June 2020 / Accepted: 16 June 2020 / Published online: 6 July 2020

(c) The Author(s) 2020. This article is published with open access

\begin{abstract}
Our recent findings link the apolipoprotein E4 (ApoE4)-specific changes in brain phosphoinositol biphosphate (PIP 2 ) homeostasis to the susceptibility of developing Alzheimer's Disease (AD). In the present study, we have identified miR-195 as a top micro-RNA candidate involved in the ApoE/PIP 2 pathway using miRNA profiles in human ROSMAP datasets and mouse microarray studies. Further validation studies have demonstrated that levels of miR-195 are significantly lower in human brain tissue of $A p o E 4^{+l-}$ patients with clinical diagnosis of mild cognitive impairment (MCI) or early AD when compared to ApoE4 ${ }^{-1-}$ subjects. In addition, brain miR-195 levels are reduced along with disease progression from normal aging to early $\mathrm{AD}$, and cerebrospinal fluid (CSF) miR-195 levels of MCI subjects are positively correlated with cognitive performances as measured by mini-mental status examination (MMSE) and negatively correlated with CSF tau levels, suggesting the involvement of miR-195 in early development of AD with a potential impact on cognition. Similar differences in miR-195 levels are seen in ApoE4 $4^{+/+}$mouse hippocampal brain tissue and cultured neurons when compared to ApoE3 $3^{+/+}$counterparts. Over-expressing miR-195 reduces expression levels of its top predicted target synaptojanin 1 (synj1), a brain PIP $_{2}$-degrading enzyme. Furthermore, elevating miR-195 ameliorates cognitive deficits, amyloid plaque burden, and tau hyper-phosphorylation in $A p o E 4^{+/+}$mice. In addition, elevating miR-195 rescues AD-related lysosomal defects in inducible pluripotent stem cells (iPSCs)-derived brain cells of ApoE4 ${ }^{+/+}$AD subjects while inhibiting miR-195 exacerbates these phenotypes. Together, our data uncover a novel regulatory mechanism of miR-195 targeted at ApoE4associated brain $\mathrm{PIP}_{2}$ dyshomeostasis, cognitive deficits, and $\mathrm{AD}$ pathology.
\end{abstract}

These authors contributed equally: Jiqing Cao, Min Huang

Supplementary information The online version of this article (https:// doi.org/10.1038/s41380-020-0824-3) contains supplementary material, which is available to authorized users.

Dongming Cai

dongming.cai@mssm.edu

1 James J Peters VA Medical Center, Research \& Development, Bronx, NY 10468, USA

2 Department of Neurology, Icahn School of Medicine at Mount Sinai, New York, NY 10029, USA

3 Department of Genetics and Genomic Sciences, Icahn School of Medicine at Mount Sinai, New York, NY 10029, USA

4 Mount Sinai Center for Transformative Disease Modeling, Icahn School of Medicine at Mount Sinai, New York, NY 10029, USA

5 Cornell University, Ithaca, NY 14850, USA

\section{Introduction}

The apolipoprotein E4 (ApoE4) allele has been identified as a major risk factor for Alzheimer's Disease (AD) [1]. Numerous studies suggest that ApoE4 effects $\mathrm{A} \beta$ clearance [2-7], neurofibrillary tangle burden [8-11],

6 Department of Psychiatry, Icahn School of Medicine at Mount Sinai, New York, NY 10029, USA

7 Alzheimer Disease Rsearch Center, Icahn School of Medicine at Mount Sinai, New York, NY 10029, USA

8 Department of Neuroscience, Icahn School of Medicine at Mount Sinai, New York, NY 10029, USA

9 Ronald M. Loeb Center for Alzheimer's disease, Icahn School of Medicine at Mount Sinai, New York, NY 10029, USA

10 James J Peters VA Medical Center, MIRECC, Bronx, NY 10468, USA 
synaptogenesis, and synaptic plasticity [12-15], glial activation and neuro-inflammation [16-18]. Moreover, ApoE proteins play central roles in lipid metabolism and neuronal homeostasis [19]. Prior studies reveal distinct alterations in brain membrane phospholipid composition, metabolism, and selected enzyme activities in postmortem AD brains [20-24], that can be exacerbated by ApoE4 [25]. We have reported that $A p o E$ proteins are critical determinants of brain phosphoinositol biphosphate $\left(\mathrm{PIP}_{2}\right)$ homeostasis, and the ApoE4 isoform is dysfunctional in this process contributing to the increased susceptibility of cognitive decline in AD [26]. We have shown that brain $\mathrm{PIP}_{2}$ levels are lower in ApoE4 brains and neurons due to the increased expression of a $\mathrm{PIP}_{2}$-dergading enzyme, synaptojanin 1 (synj1) [26].

The functional roles of $\mathrm{PIP}_{2}$ and synj1 have been implicated in $\mathrm{AD}$ pathogenesis by our laboratory and others [26-32]. For example, increased expression of synj1 is linked to early endosome enlargement [33], and ApoE4-associated cognitive deficits in AD [26]. The reduction of synj1 provides several beneficial effects in $\mathrm{AD}$ such as accelerating $\mathrm{A} \beta$ clearance via the lysosomal degradation pathway [30], ameliorating mild traumatic brain injury (TBI)-induced elevation in tau hyperphosphorylation [31], and rescuing ApoE4-associated cognitive impairments [26]. However, molecular signaling mechanisms that link ApoE4 with brain $\mathrm{PIP}_{2} /$ synj1 pathways and impact on cognitive function remain elusive. We have previously shown that increased synj1 levels in ApoE4 brains are partially due to a failure in efficient synj1 mRNA degradation [26]. mRNA stability is often regulated by micro-RNA (miRNA) binding to $3^{\prime}$ UTR regions of mRNA [34]. We postulate that brain synj1 expression may be differentially regulated by ApoE isoforms through miRNA modulation.

Several miRNAs have been previously implicated in various AD processes [35]. Moreover, changes in various brain miRNA levels between AD subjects and normal-aged controls have been reported $[36,37]$. Therefore, in the present study, we leveraged the existing miRNA datasets from the Religious Orders Study and the Rush Memory and Aging Project (ROSMAP) [38, 39], in combination with miRNA array studies we performed in $\mathrm{ApoE4}^{+}$and $\mathrm{ApoE4} 4^{-}$cortical neurons. We have identified a miRNA, miR-195, as a top candidate that is differentially expressed between $\mathrm{ApoE4}^{+}$ and $\mathrm{ApoE4}^{-}$carriers and targeted at synjl mRNA as predicted by multiple bioinformatics databases including mirDB [40, 41]. The changes in miR-195 levels are further validated using postmortem human and mouse brain tissue as well as cultured neurons. Furthermore, we have characterized a regulatory role of miR-195 in ApoE4-associated brain $\mathrm{PIP}_{2}$ dyshomeostasis, cognitive deficits, and AD pathology.

\section{Materials and methods}

\section{Human miRNA expression profile and data preprocessing}

The miRNA expression profile was downloaded from the ROSMAP study (Synapse https://doi.org/10.7303/ syn3388564). miRNAs that had a call rate less than $95 \%$ and an absolute value of lower than 15 in $<50 \%$ of the samples were removed. miRNA expression values were normalized using a variance stabilization normalization method. Cartridges were specified as batches and were corrected with the Combat function in the $\mathrm{R}$ package sva (V3.20.0). The data pre-processing resulted in 309 miRNAs in 511 samples. Pre-processed RNA-seq FPKM gene expression abundance data were also downloaded from the ROSMAP study (Synapse https://doi.org/10.7303/ syn3388564). Genes with at least 1 FPKM in at least $10 \%$ of the samples were selected, and data were then corrected for confounding factors including batch, PMI, and RIN scores. The pre-processed gene expression profile contains 16,235 genes and 619 samples.

\section{Differential expression and miRNA-gene correlation analysis}

Differential expression analysis was performed on all miRNAs between $111 \mathrm{APOE}^{-/-}$( $\left.83 / 3\right)$ and $24 \mathrm{APOE}^{+/-}(\varepsilon 3 / 4)$ carriers using the R package limma (V3.34.0) [42]. Multiple tests were adjusted using the Benjamini-Hochberg's (BH) FDR method. Correlation analysis was performed between miRNAs and genes using spearman's correlation test. The miRNA-gene correlation was also examined in each of the subgroups of $\mathrm{AD}$ diagnosis, sex, and $A P O E$ genotype.

\section{miRNA array studies of mouse primary neuron samples}

Embryonic 17 days old $A p o E^{-l-}$ cortical neurons were cultured for 7 days in vitro in the presence of conditioned media derived from ApoE3 and ApoE4 primary astrocyte cultures as described [26, 43] ( $N=3$ /group). miRNAs were extracted using miRCURY extraction kits (Exiqon Inc.) and then labeled using miRCURY LNA microRNA Hi-Power Labeling kit, Hy3/Hy5, and hybridized on the miRCURY LNA microRNA Array. The quality assessment using control spike-in oligo nucleotides produced signals in expected range indicated successful labeling. Following normalization of quantified signals after background correction using global Lowess regression algorithm, unsupervised and supervised data analysis were performed. The microRNA profiling identified a subset of microRNAs that 
are differentially expressed in the ApoE3 versus ApoE4treated neurons.

\section{miRNA target prediction}

Targets of the miRNAs were predicted with the $\mathrm{R}$ package multiMiR (V2.2.0), which is a miRNA-target interaction database complying nearly 50 million human and mouse data from 14 different databases [44].

\section{Human brain and CSF sample preparation}

Equal amounts of postmortem human parietal cortex brain tissues $(50 \mu \mathrm{g}$ by net weight) from the NIH brain and tissue repository (NBTR), as well as cerebrospinal fluid (1 $\mathrm{ml}$ by net weight) from mild cognitive impairment (MCI) subjects enrolled in the James J Peters VAMC study ("Markers of Transition to AD in the Veterans with MCI") and Icahn School of Medicine at Mount Sinai (ISMMS) AD Research Center participants were used for studies. The experimental procedures involving human sample handling were approved by the appropriate committees at James J Peters VA Medical Center (JJP VAMC) and ISMMS. Patient demographic information with $\mathrm{N} /$ group provided in Table S1.

\section{Animal models}

Human $A p o E 4^{+/+}$or $A p o E 3^{+/+}$knock-in (KI) mouse models without [45-47] or with 5xFAD background [48, 49] were genotyped as described [50]. All animal experiments were performed in accordance with $\mathrm{NIH}$ guidelines and were approved by the JJPVAMC and ISMMS Institutional Animal Care and Use Committees (IACUC). Sex as a biological variable was taken into considerations with inclusion of both male and female mice in all experiments.

\section{Phospholipid analysis}

Samples were subjected for lipid extraction, followed by the quantification by anion-exchange HPLC as described previously [30, 51].

\section{Mouse Neuronal and human iPSC culture}

Primary cortical neurons were cultured as described [26, 43], before being fixed and stained for confocal microscopy analysis (Zeiss LSM) [52]. In some conditions, bovine serum albumin (BSA) or receptor-associated protein (RAP) was included in the cultured media ( $N=3$ /group). Alternatively, cultured neurons were transfected with adeno-associated virus 2 (AAV2)-containing miR-195 or
miR-374 or scramble controls for 5 days before subjected to analysis. The ApoE4 $4^{+/+}$and $A p o E 3^{+/+}$iPSCs were differentiated into neural progenitor cells (NPCs) by dual SMAD inhibition followed by neural rosette selection and forebrain-specific patterning by $20 \mathrm{ng} / \mathrm{ml} \mathrm{FGF2} \mathrm{exposure} \mathrm{as}$ described [53, 54]. These NPCs were purified by MACS for CD271 ${ }^{-} / \mathrm{CD}_{133^{+}}{ }^{[53]}$ and differentiated to cortical neurons $[55,56]$ and a homogeneous population of astrocytes $[54,57]$ before subjected to viral transfection and confocal microscopy analysis. Alternatively, mouse cortical neurons derived from $A p o E 4^{+/+}$and $A p o E 3^{+/+} \mathrm{KI}$ mice with synj $1^{+/+}$or synj1 $1^{-/-}$genotypes were co-cultured with ApoE4 $4^{+/+}$and ApoE3 $3^{+/+}$iPSC-derived pure astrocytes. In some experiments, cultured neurons were transfected with AAV2-containing miR-195, scramble controls, or miR195 inhibitors that specifically prevent miR-195 binding to its target mRNA before co-culturing with iPSC-derived astrocytes. Cultured iPSC-derived neurons and astrocytes were then incubated with lysotracker red for various time periods before fixation and double-stained for pTau and a nuclear marker DAPI (blue) for confocal microscopy analysis (Zeiss). $N=4-5 /$ condition.

\section{Stereotaxic injection and behavior studies}

Eight to nine weeks old male and female $A p o E 3^{+/+}$and ApoE4 $4^{+/+}$KI mice without $5 \times$ FAD background $(N=$ 19-23/group), or with 5xFAD background $(N=15-17 /$ group) were placed in the stereotaxic apparatus with AAV2 or scramble virus administered into the dorsal CA1 regions of bilateral hippocampal brain regions using pressure injection as described [58, 59]. Injection volumes $(0.5-2.0 \mu \mathrm{l})$ were delivered over $10 \mathrm{~min}$ to avoid tissue damage. Six to nine months after viral delivery, mice were tested with the NOR task as described [26, 60, 61]. Mice were randomized for genotype and sex, and blinded throughout the behavior data collection and analysis, surgical manipulations, and sample collection followed NIH practice guidelines. Animals were excluded from behavior analysis if the total exploration time was less than $4 \mathrm{~s}$ or if they had an illness that prevented them from reliably completing the behavior tests.

\section{Brain and neuronal sample preparation and biochemical analysis}

Snap-frozen mouse hemi-brains or cultured neurons were harvested in lysis buffer [62] and processed via step-wise solubilization [62, 63], followed by SDS-PAGE to determine levels of synj1, dyn1, holoAPP, and CTFs. Levels of $\mathrm{A} \beta_{42}, \mathrm{~A} \beta_{40}$, pTau, Tau and ApoE were determined using high-sensitive ELISA kits. Some tissue was used for miRNA and RNA extraction followed by qPCR and RNA- 
seq analysis. Some animals underwent perfusion followed by brain tissue section for immunohistochemical staining of amyloid plaque, synj1 and pTau.

\section{Differential gene expression analysis for miR-195 treated mice}

The RNA-seq samples collected from mouse brains were profiled on the Illumina HiSeq platform. Quality control of generated reads was performed using FASTQC (0.11.8). The raw sequencing reads were aligned to the GRCm38 mouse genome (release 95) using star aligner (V2.5.0b). Following the read alignment, gene expression was quantified at the gene level based on Ensembl gene model GRCm38.95 using FeatureCounts [64]. Genes with at least one count per million in all samples were considered as expressed and hence retained for further analysis. The trimmed mean of M-values normalization method [65] was used to adjust for sequencing library size differences. Differential expression analysis was then performed on the quality controlled and normalized gene expression data using the $\mathrm{R}$ package limma (V3.34.0). The comparisons were carried out between miR195-treated and scramble control samples stratified by sex and APOE genotype. Multiple tests were adjusted using the BH FDR method.

\section{Functional enrichment analysis}

The functional enrichment analysis was carried out for genes significantly correlated with miRNAs in human ROSMAP dataset, predicted target genes of each miRNA of interest, and differentially expressed genes identified from miR195-treated mouse RNA-seq dataset. These genes were queried against the molecular signatures database (MSigDB v6.1) using Fisher's Exact Test and gene set enrichment analysis (GSEA) [66].

\section{Antibodies and reagents}

The anti-synj1 (rabbit polyclonal Ab, Novus, RRID: AB_11047653), anti-pTau AT8 and Tau-5 (ThermoFisher, RRID: AB_223647 and 10980631), anti-Rab5 (Santa Cruz Biotechnology, RRID:AB_628191), anti- $\beta$ actin and tubulin (Santa Cruz Biotechnology, RRID:AB_476697 and 477498), anti-holoAPP MAB348 and 6E10 (Millipore RRID:AB_94882 and 564201), anti-beta-Amyloid (Cell Signaling Technology, RRID: AB_2056585), anti-MAP2 (Abcam, RRID:AB_297885), anti-dynamin clone 41 for (BD bioscience; RRID:AB_3976413), anti-mouse and rabbit HRP (ThermoFisher, RRID:AB_2556542 and 2540618), Texas-Red and Alexa 555 conjugated anti-mouse and rabbit IgG (ThermoFisher, RRID:AB_10374713, 10983944, 2535987 and 1090271) were purchased. AAV2- containing miR-195, miR-374, scramble controls and miR195 inhibitors were generated and obtained from ABM Inc. with detailed sequence information available (Am00100, Amm1017200 and Amm3026700). The miRNA extraction kit and qPCR probes for specific miRNAs were purchased from Exiqon Inc. The qPCR probes for actin (Hs1060665_g1), synj1 (Hs00953234_m1 and Mm01210539_m1), gapdh (Mm99999915_g1), RNU6B (NR_002752), $18 \mathrm{~s}$ and $45 \mathrm{~s}$ (4331182, Mm03928990_g1) were also purchased from ThermoFisher.

\section{Statistical analysis}

The sample sizes of each experiment were chosen based on power calculations derived from previous similar studies, which allowed us to determine group sizes needed to achieve statistically significant results. All experiments including controls were performed in randomly assigned groups. Sample collection and data analysis followed NIH practice guidelines. Experimenters were blinded to the experimental condition of the animal while conducting experiments. The conditions were revealed after quantification was completed. Levels of miR-195 and mi-374 were normalized to U6 and RNU6B (internal controls) while synj1 mRNA normalized to GAPDH and $18 \mathrm{~s}$, and then expressed as $\log _{2}$ fold of changes when compared to controls. Levels of synj1, dyn1, pTau, Tau, and holoAPP were normalized to $\beta$-actin levels and expressed as a percentage of the control. Absolute $A \beta_{42}, A \beta_{40}$, pTau, Tau, and ApoE concentrations were quantitatively determined by ELISA and expressed as a percentage of the control. Independentsamples $t$-tests were used to determine significant mean differences (the threshold for significance sets at $p<0.05$ ). ANOVA with post-hoc tests were used to determine group differences for multiple comparisons. Pearson correlation coefficients were calculated to determine the linear relationship between the two variables. Equality of variance was checked for all statistical comparisons. When independent-samples $t$-tests were used and equality of variances of compared groups were not the same, the Welch's corrections were applied. All statistical analysis was performed using Prism 8.0.

\section{Results}

\section{MiR-195 is identified as a top candidate miRNA involved in APOE-regulated synj1 expression}

First, we performed differential expression analysis between $A p o E 4^{-/-}(\varepsilon 3 / \varepsilon 3 ; N=111)$ and $A p o E 4^{+/-}(\varepsilon 3 / \varepsilon 4 ; N=24)$ carriers on human miRNA profiles in ROSMAP datasets. We identified 16 significantly differentially expressed 




Fig. 1 MiR-195 is identified as a top miRNA candidate involved in APOE-regulated synj1 expression. a Venn diagram shows miR-195 as the only miRNA in common shared among 4 groups: miRNAs differentially expressed between $\mathrm{ApoE4}^{+}$and $\mathrm{ApoE4^{- }}$ carriers in the human ROSMAP dataset, miRNAs differentially expressed between $\mathrm{ApoE}^{+}$and $\mathrm{ApoE4}^{-}$in the mouse miRNA array studies, miRNAs negatively correlated with synj1 mRNA in ROSMAP, and miRNAs predicted to target at synj1 mRNA by multiMiR database. Numbers of

miRNAs ( $p<0.05), 12$ of which with reduced expression in the $A p o E 4^{+/-}$carriers (Fig. 1a). In parallel, we performed miRNA array studies of $A p o E^{-/-}$hippocampal neurons treated with ApoE3 or ApoE4-conditioned media (CM) (Fig. 1a). We identified 30 significantly differentially expressed miRNAs $(p<0.05), 15$ of which with reduced expression in the ApoE4-treated conditions (Fig. S1A). Among these miRNAs, miR-195 is the only differentially expressed miRNA between $A p o E 4^{+}$and $A p o E 4^{-}$conditions that is commonly shared between human and mouse datasets (hsa-miR-195-5p and mmu-miR-195a-5p; Fig. 1b). Another miRNA, miR-155 is also identified in both human and mouse datasets (hsa-miR-155 and mmu-miR-155-5p) but in opposite trends (higher in human $\mathrm{ApoES}^{+-}$carriers and lower in mouse ApoE4-treated conditions; Fig. S1B).

Next, we performed prediction of miRNAs that putatively bind with synj $1 \mathrm{mRNA}$ using 14 compiled multiMiR database [44]. From a total of 392 (human)/194 (mouse) miRNAs targeted at synj1 mRNA (Fig. 1a), miR-195 was predicted as a top candidate in the human mirDB database [40, 41] (predicting score: 99.9/100). MiR-195 was also predicted as a top candidate miRNA targeted at synjl in several other databases (Fig. S1C) such as elmmo (predicting score: $0.80 / 1$ ), and diana_micro (predicting score: $0.79 / 1$ ).

Furthermore, we examined the correlation between synj1 mRNA and all miRNAs in ROSMAP dataset using Spearman's correlation test. MiR-195 is negatively correlated with synj1 mRNA levels in all human subjects (Fig. 1c: $r=$ $-0.115, \quad p=0.0093)$. Similarly, negative correlations between miR-195 and synjl mRNA levels are seen in all female subjects $(r=-0.167, p=0.0026)$ and in $A p o E 3^{+/+}$
miRNAs overlapping among subgroups are indicated (red numbers). b Log Fold of changes (LogFC) and $p$ values of differences in miR-195 levels between $A p o E 4^{+}$and $A p o E 4^{-}$carriers, between female $A p o E 4^{+}$ and $A p o E 4^{-}$carriers in ROSMAP dataset, as well as differences in miR-195 levels between mouse $A p o E 4^{+}$and $A p o E 4^{-}$treated neurons. c Analysis of correlation between miR-195 and synj1 mRNA in human subjects of the ROSMAP database.

carriers $(r=-0.127, p=0.027)$. A negative correlation trend can be seen in $A p o E 4^{+-}$carriers as well but with no statistical significance $(r=-0.178, p=0.057)$, suggesting a possible weakened or perturbed network regulation between miR-195 and synj1 in the presence of ApoE4 allele. Separately, miR-374b also showed negative correlation with synj1 mRNA $(r=-0.09, p=0.04)$. In addition, mmumiR-374b-5p is differentially expressed between ApoE3 and ApoE4-treated conditions but the differences in hsa-miR-374-5p levels between human $A p o E 4^{+/-}$and ApoE $4^{-1-}$ carriers are not statistically significant (Fig. S1B, $p=0.188$ ).

To better understand the molecular pathways miR-195 regulates, we investigated the functions of predicted miR195 targeted genes and those significantly correlated with miR-195 in the ROSMAP dataset. The top enriched functions for target genes and genes negatively correlated with miR-195 include regulation of neuronal and synaptic function, neurogenesis, and differentiation, while functions of genes positively correlated with miR-195 are enriched in the circulatory system and vasculature development (Table S2).

Together, these results support a role of miR-195 as a top candidate miRNA in regulating the ApoE-synj1-PIP 2 pathways.

\section{Reduction of brain miR-195 levels is associated with ApoE4 genotype, disease progression and cognitive decline}

To validate differential expression patterns of miR-195 between $\mathrm{ApoE}^{+/-}$and $\mathrm{ApoE4^{-/- }}$ carriers, we next 
A)



C)



Fig. 2 Reduction of brain miR-195 levels in human brain and CSF samples is associated with ApoE4 genotype, disease progression, and cognitive decline. a Amounts of miR-195 (presented as $\log _{2}$ fold changes) in human parietal cortex tissue of $\mathrm{ApoE4}^{+/-}$subjects


group; $\log _{2}$ FC fold of changes: ApoE4 $4^{-l-} 0.054 \pm 0.113$ versus ApoE4 $4^{+/-}-0.570 \pm 0.178, \quad * * p<0.01$ with independent-samples $t$-tests. b Pattern of reduction in miR-195 levels (presented as $\log _{2}$

examined miR-195 levels in human brain tissue and CSF samples (subject demographic information provided in Table S1). We found that miR-195 levels were reduced in parietal cortex tissues derived from ApoE4 $4^{+/-}$mild cognitive impairment (MCI) and early AD subjects with clinical dementia rating (CDR) scores between 0.5 and 1 compared to levels in $A p o E 4^{-l-}$ donors (Fig. 2a). Interestingly, we observed a pattern of reduction in miR-195 levels along with disease progression from normal aging to MCI and early AD (Fig. 2b), similar to what we previously seen with $\mathrm{PIP}_{2}$ and phosphoinositol (PI) changes in early AD development [26]. We also found a significant reduction in miR195 in female subjects compared to male subjects (Fig. S2A), with differences also noted between male ApoE $4^{-1-}$ versus female ApoE $4^{+/-}$subjects. Consistently, we observed a reciprocal elevation of synj1 mRNA levels in $A p o E 4^{+/-}$subjects when compared to levels in ApoE4 $4^{-/-}$
B) $\operatorname{miR} 195$



D)

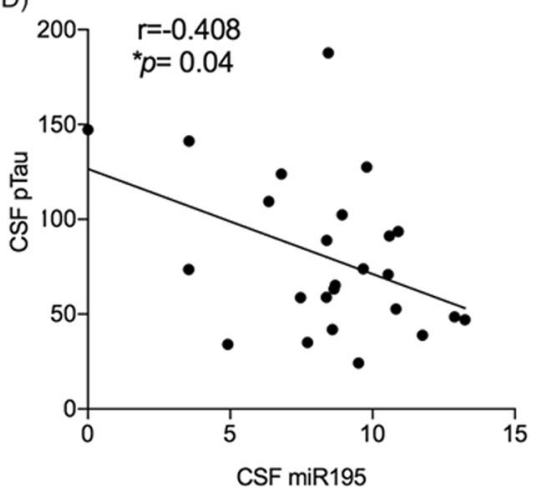

fold changes) along with $\mathrm{AD}$ disease progression from normal aging to MCI and early AD. $N=12-19$ /group; $\log _{2} \mathrm{FC}: 1.626 \pm 0.696$ in CDR 0 subjects versus $0.242 \pm 0.104$ in CDR 0.5 MCI patients; versus $-0.663 \pm 0.135$ in $\mathrm{CDR} 1 \mathrm{AD}$ subjects; $* p<0.05$, **** $p<0.0001$ with ANOVA tests. c Positive correlation between CSF miR-195 levels and MMSE scores $(r=0.455, p=0.029 ; N=23)$. d Negative correlation between CSF miR-195 and pTau levels $(r=-0.408, p=$ $0.04 ; N=23)$.

subjects (Fig. S2B). A positive correlation was noted between brain miR-195 and $\mathrm{PIP}_{2}$ levels in $\mathrm{ApoE4}^{-1-}$ carriers with CDR $0.5-1$, and a positive correlation trend was seen in CDR 0-1 subjects regardless of ApoE genotypes (Fig. S2C). No correlation was seen between brain miR-195 and other phospholipid species, e.g., PI and phosphoinositol phosphate (PIP). No correlation was seen between brain miR-195 and other variables such as post-mortem interval (PMI) and age. A negative correlation was observed between brain miR195 and another known target of miR195, beta-secretase 1 (BACE-1) [67] expression in the CDR 0.5-1 cohort (Fig. S2D). However, we did not observe any correlation between miR-195 and $\mathrm{A} \beta$ levels (data not shown).

There were statistically significant differences in human


(hsa-miR374-5p; Fig. S3A). However, there were no 
A)



C)



B) $\operatorname{miR} 195$



D)

$$
\text { synj1 protein }
$$



Fig. 3 MiR-195 expression is reduced in hippocampal brain tissue and cultured primary neurons of ApoE4 mice; modulating miR195 levels regulates synaptojanin 1 expression. a Levels of miR-195 were reduced in 12-month old ApoE4 hippocampal brain tissue $\left(\log _{2} \mathrm{FC}:-0.283 \pm 0.069\right)$ when compared to those in ApoE3 mice $\left(\log _{2}\right.$ FC: $\left.-0.036 \pm 0.034\right) . N=11-13$ /group with both males and females; ${ }^{* *} p=0.0096$ with ANOVA tests. A nominal reduction in miR-195 levels was seen in $A p o E^{-1-}$ brains with no statistical significance $\left(\log _{2} \mathrm{FC}:-0.125 \pm 0.067, p=0.48\right)$. b Levels of miR-195 in ApoE ${ }^{-1-}$ neurons treated with ApoE4-CM were reduced $\left(\log _{2} \mathrm{FC}=\right.$ $-0.314 \pm 0.073$, when compared to levels of those treated with ApoE3-CM $\left(\log _{2}\right.$ FC: $\left.0.184 \pm 0.094\right)$. $N=5 /$ group; $* * p=0.003$ with independent-samples $t$-tests. c Differences in miR-195 expression

significant changes in miR-374 levels along the disease progression except a transient elevation at MCI stage (Fig. S3A). Consistently with ROSMAP data (Fig. S1B), we also observed statistically significant differences in human miR-155 levels with higher levels in ApoE4 $4^{+-}$ subjects (hsa-miR155-5p; Fig. S3B). No significant differences were seen in miR-155 levels along disease progression (data not shown). Moreover, no significant levels between ApoE3-CM and ApoE4-CM treated neurons were abolished in the presence of RAP. The treatment of RAP in the presence of ApoE3-CM led to a reduction in miR-195 levels $\left(\log _{2} \mathrm{FC}\right.$ : $-1.648 \pm 0.125 ; p<0.0001)$, whereas in ApoE4-CM treated conditions, miR-195 levels were much lower at baseline with a trend of improvement in the presence of RAP treatment (ApoE4 CM + BSA $\log _{2} \mathrm{FC}:-3.193 \pm 0.144$ versus ApoE4 CM + RAP $\log _{2} \mathrm{FC}:-2.678 \pm$ $0.054 ; \quad p=0.052$ ). $\quad N=3$ /group; $\quad * * * * p<0.0001$ by One-Way ANOVA tests. d Synj1 protein levels were reduced with miR-195 over-expression but not miR-374 over-expression in $A p o E^{-1-}$ hippocampal neurons in the presence of ApoE4-CM. $N=4$ /group; synj1 levels with miR-195: $62.87 \pm 4.48 \%$ of controls, $* * p=0.001$; with miR-374: $102.4 \pm 7.77 \%$ of controls, $p=0.93$.

differences were seen in miR-195 or miR-374 levels between $A p o E 4^{+/-}$and $A p o E 4^{-/-}$subjects of normal aging or advanced AD either (CDR 0 or 3 and above; data not shown), suggesting the functional involvement of miR-195 in early disease development and acceleration by ApoE4 genotype.

Using cerebrospinal fluid (CSF) samples from a cohort of MCI subjects with CDR 0.5 (MCI defined by clinical 
A)

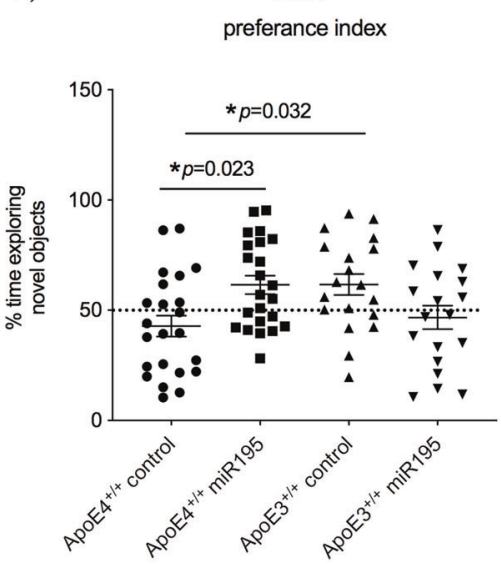

C)

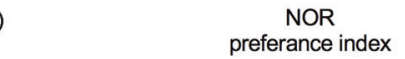

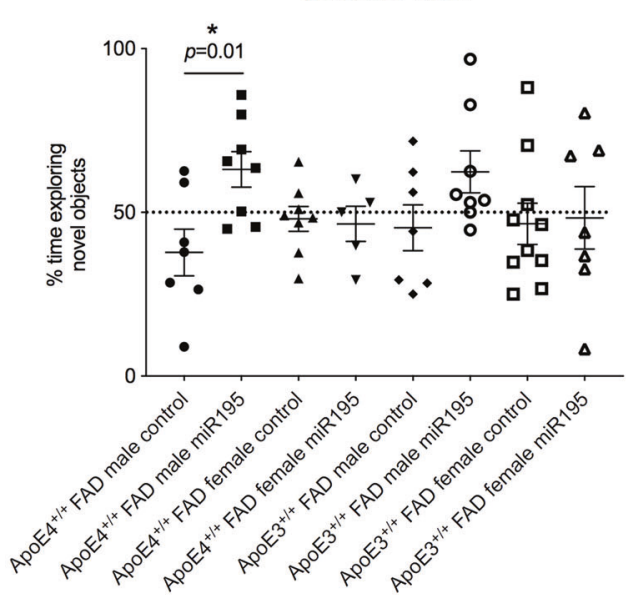

E)



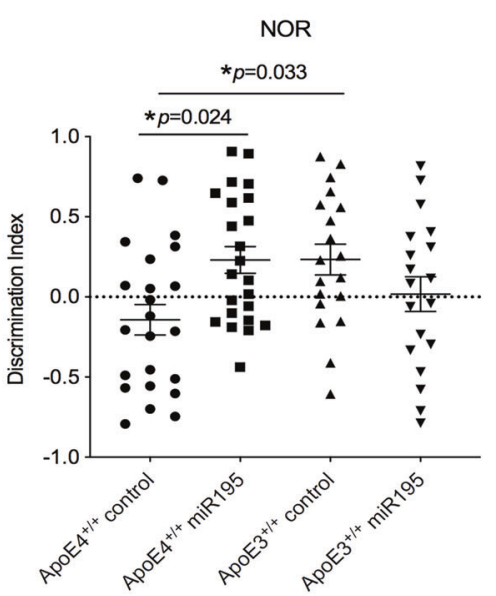

B)

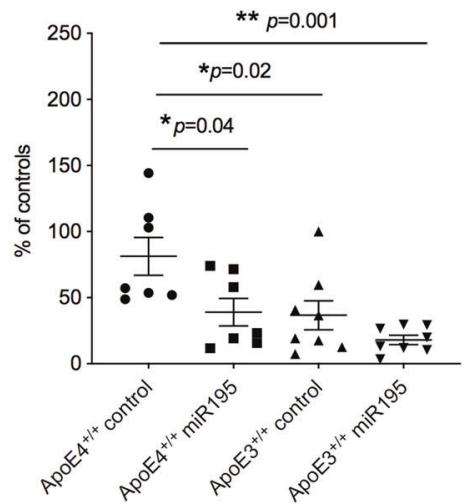

D)

pTau
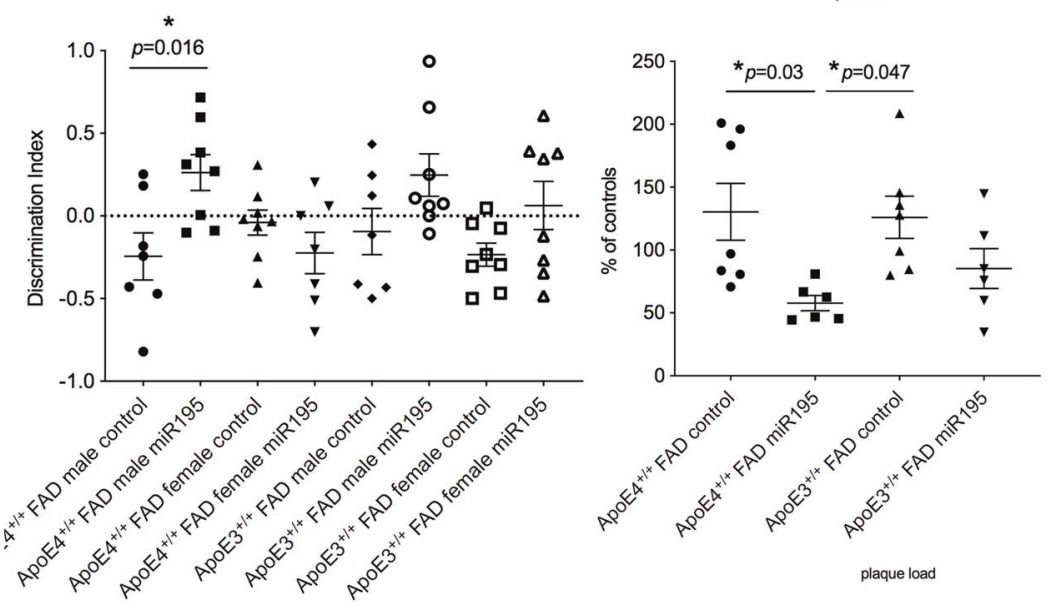

F)
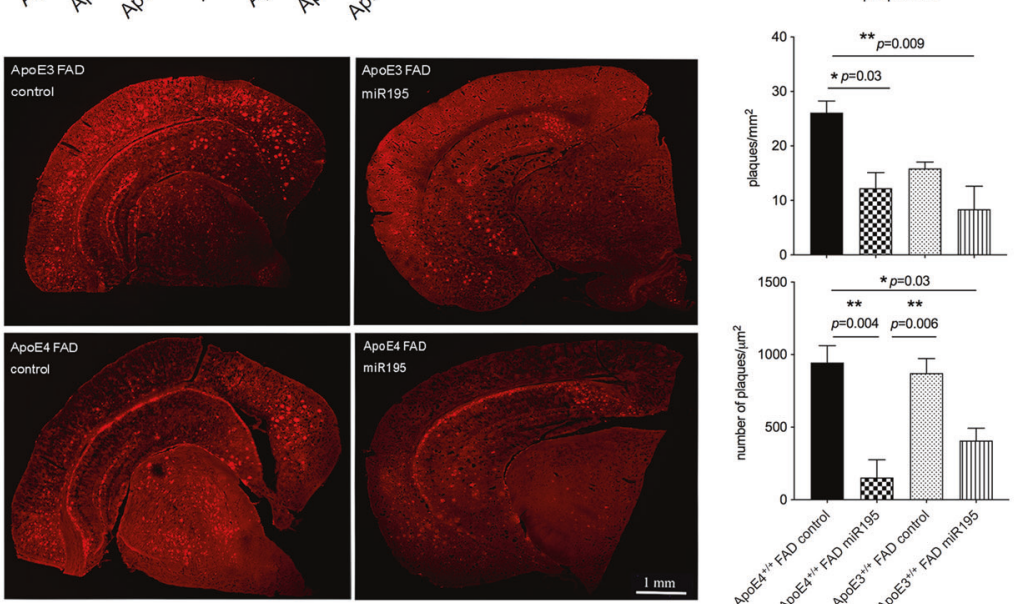

examination and neuropsychological assessments), we found that CSF miR-195 levels were positively correlated with cognitive performance measured by mini-mental status examination (MMSE; Fig. 2c), and negatively correlated with pTau levels (Fig. 2d). While CSF PIP 2 levels were below the detectable range, a positive correlation was seen between CSF cardiolipin and miR-195 (Fig. S3C), suggesting a potential involvement of miR-195 in mitochondrial function [68, 69].

Together, our results suggest that reduction of brain and CSF miR-195 levels is associated with ApoE4 genotype, cognitive decline, and tau pathology during early AD development. 
Fig. 4 Over-expression of miR-195 rescues cognitive deficits and ameliorates AD-associated pathologies in ApoE4 mouse models. a Preference index $=$ (time exploring novel object)/(time exploring novel object + time exploring familiar object) and discrimination index $=$ (time exploring novel object- time exploring familiar object)/ (time exploring novel object + time exploring familiar object) in 4

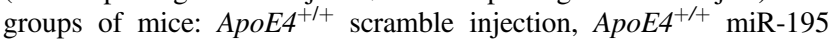
injection, ApoE3 ${ }^{+/+}$scramble injection, and $A p o E 3^{+/+}$miR-195 injection. $N=19-23 /$ group with both males and females; $* p<0.05$ with ANOVA tests. b Levels of pTau in KI mouse hippocampus. $N=$ 8/group with both males and females; $* p<0.05$; $* * p<0.01$ with ANOVA tests. c Preference index and discrimination index in 8

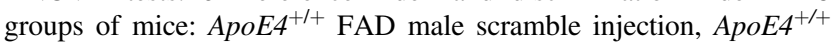
FAD male miR-195 injection, ApoE4 ${ }^{+/+}$FAD female scramble

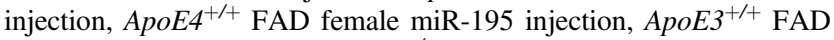
male scramble injection, ApoE $3^{+/+}$FAD male miR-195 injection, $A p o E 3^{+/+}$FAD female scramble injection, and $A p o E 3^{+/+}$FAD female miR-195 injection. $N=6-10 /$ group; $* p<0.05$ with ANOVA tests. Levels of $\mathbf{d}$ pTau and $\mathbf{e}$ oligomer $\mathrm{A} \beta_{42}$ in EFAD mouse hippocampus. $N=6 /$ group with both males and females; $* p<0.05 ; * * * p<0.001$; $* * * * p<0.00001$ with ANOVA tests. f Amyloid plaque burden in EFAD mouse hippocampus. A representative image of brain section is shown. Amyloid plaque load is quantified by density measured as area of plaques per $\mathrm{mm}^{2}$ of brain region, as well as total numbers of plaques $/ \mu \mathrm{m}^{2}$ in 4 groups of mice: $A p o E 4^{+/+} \mathrm{FAD}$ scramble injection, ApoE $4^{+/+}$FAD miR-195 injection, ApoE $3^{+/+}$FAD scramble injection, and $A p o E 3^{+/+}$FAD miR-195 injection. $N=3$ /group; $* p<0.05$; $* * p<$ 0.01 with ANOVA tests.

\section{MiR-195 expression is reduced in ApoE4 mouse brains and cultured neurons}

Next, we investigated if differences in miR-195 levels can be recapitulated in mouse models and primary neurons. We found that the levels of miR-195 were lower in 12month old $\mathrm{ApoE}^{+/+}$mouse brains compared to $\mathrm{ApoE3^{+/+ }}$ mice (Fig. 3a). A nominal reduction in miR-195 levels was seen in $A p o E^{-1-}$ brains. Similarly, miR-374 was decreased in $A p o E 4^{+/+}$mouse brains when compared to ApoE3 conditions (Fig. S4A) with a nominal reduction in $A p o E^{-1-}$ mice. In cultured $A p o E^{-1-}$ hippocampal neurons, levels of miR-195 were consistently lower with ApoE4 CM from astrocytes compared to those with ApoE3 CM (Fig. 3b). A nominal difference was noted in miR-374 levels in neurons treated with ApoE4 CM when compared to those treated with ApoE3 CM (Fig. S4A) but failed to achieve statistical significance due to large variations among samples.

Interestingly, treatment of ApoE-receptor-associated protein (RAP), an inhibitor of $A p o E$ receptors [70, 71] abolished differential expression patterns of miR-195 relative to control (BSA: bovine serum albumin). The RAP treatment in the presence of ApoE3-CM led to a reduction in miR-195 levels (Fig. 3c), whereas in ApoE4-CM treated conditions, miR-195 levels were much lower at baseline with a trend of improvement following RAP treatment. These results suggest that astrocyte-derived ApoE likely binds to $A p o E$ receptors on neurons leading to changes in neuronal miR-195 and ApoE4 exhibits loss-of-function effects on neuronal miR-195 expression.

We then determined if upregulation of miR-195 in ApoE4 conditions could modulate expression levels of its predicted target gene, synj1. Over-expression of miR-195 but not miR-374 significantly reduced synj1 protein levels in $A p o E^{-/-}$neurons treated with ApoE4 CM (Fig. 3d; synj1 levels with miR-195: $62.87 \pm 4.48 \%$ of controls, $p=0.001$; with miR-374: $102.4 \pm 7.77 \%$ of controls, $p=0.93$ ). No changes were seen in expression levels of another endocytic adapter protein dynamin 1 (dyn1; Fig. S4B), suggesting a specific effect of miR-195 on synj1 expression. Similarly, miR-195 over-expression in $\mathrm{ApoE3}^{+/+}$or $\mathrm{ApoE4}^{+/+}$neurons resulted in synj1 expression reduction in both mRNA and protein levels (Fig. S4C and D). It should be noted that $A p o E 4^{+/+}$neurons exhibited more dramatic changes with over-expression of miR-195 in synj1 mRNA $\left(\right.$ ApoE $^{+/+} \mathrm{w}$ miR-195 $\log _{2} \mathrm{FC}:-1.084 \pm 0.035$ versus $A p o E 4^{+/+}$w miR195: $-7.751 \pm 0.043$; Fig. S4C), and protein levels $\left(\right.$ ApoE $^{+/+}$w miR-195 $70.9 \pm 21.2 \%$ versus $A p o E 4^{+/+} \mathrm{w}$ miR-195 48.0 $\pm 9.84 \%$ of controls; Fig. S4D) than $A p o E 3^{+/+}$neurons, possibly due to much lower baseline levels in $\mathrm{ApoE}^{+/+}$cells making them more sensitive to miR-195 manipulations.

\section{Over-expression of miR-195 rescues cognitive deficits and ameliorates AD-associated pathologies in ApoE4 mouse models}

We next determined if over-expressing miR-195 could rescue ApoE4-associated cognitive dysfunction in vivo using $\mathrm{ApoE4}^{+/+}$and $\mathrm{ApoE3}^{+/+} \mathrm{KI}$ mice without and with $\mathrm{AD}$ transgenic background. We previously demonstrated that male human $\mathrm{ApoE}^{+/+}$KI mice manifested memory impairments as measured by novel object recognition (NOR) tests with an inability to discriminate between novel and familiar objects [26]. Here we found that $A p o E 4^{+/+} \mathrm{KI}$ mice spent less time exploring novel objects than $A p o E 3^{+/+}$KI mice did (Fig. 4a preference index: $A p o E 4^{+/+}$versus $A p o E 3^{+/+}$ scramble controls: $42.9 \%$ versus $61.7 \%, p=0.032$ ), consistent with what we had previously observed [26]. This deficit was completely abolished by viral delivery of miR-195 bilateral hippocampi of $A p o E 4^{+/+}$KI mice (Fig. 4a, 61.5\%, $p=0.023$ when compared to $A p o E 4^{+/+}$scramble controls). However, no statistically significant differences were seen between scramble and miR-195 over-expressing $A p o E 3^{+/+}$ animals. Moreover, the discrimination index studies using the difference in exploration time for novel versus familiar object [72] showed consistent results suggesting that impaired discrimination behaviors in $A p o E 4^{+/+} \mathrm{KI}$ mice were completely rescued by miR-195 over-expression (Fig. 4a discrimination index: $A p o E 4^{+/+}$versus $A p o E 3^{+/+}$scramble controls: -0.144 versus $0.234 ; p=0.033 ; A p o E 4^{+/+}$scramble controls versus 

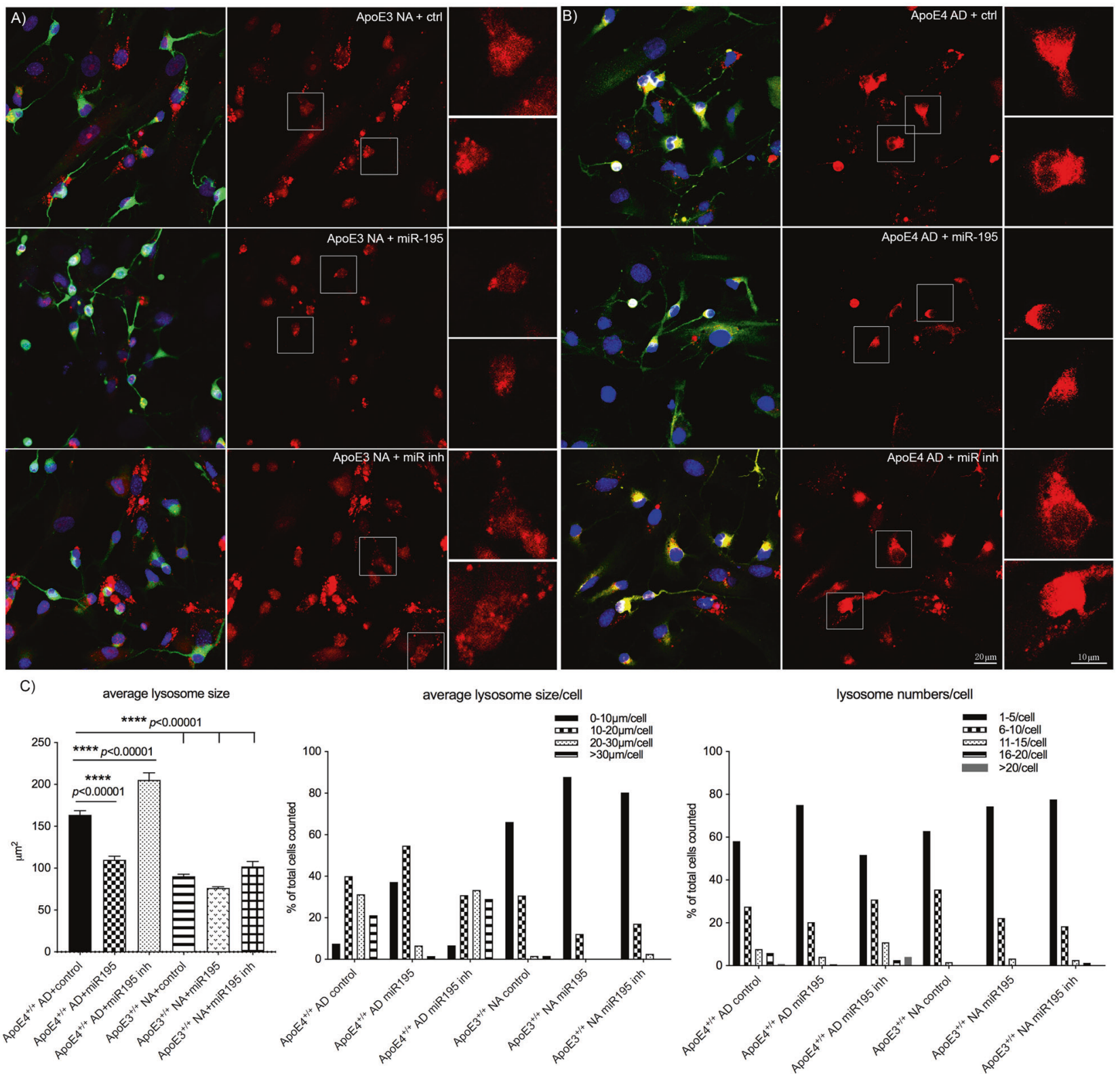

Fig. 5 Over-expression of miR-195 rescues lysosomal defects in ApoE4 iPSC-derived brain cells. Representative immunofluorescence co-staining of lysosomes (Lysotracker: red fluorescence), a neuronal marker MAP-2 (green fluorescence) and DAPI (blue fluorescence) of iPSC-derived neuron and astrocyte co-culture of (a) $\mathrm{ApoE3}^{+/+}$normal aging (NA) and (b) $\mathrm{ApoE4}^{+/+} \mathrm{AD}$ subjects with various conditions: scramble control (ctrl), miR-195, and miR-195

inhibitor (miR inh). Alternatively, $\mathrm{ApoE3}^{+/+}$and $\mathrm{ApoE}^{+/+} \mathrm{N}=3-6 /$ conditions. c Quantification of all lysosomes by size (measured by areas; $\mu \mathrm{m}^{2}$ ) of $60-90$ neurons $\left(\mathrm{MAP}-2^{+}\right.$) in each experimental condition, the distribution of lysosome sizes/cell (measured by diameters; $0-10 \mu \mathrm{m}, 10-20 \mu \mathrm{m}, 20-30 \mu \mathrm{m}$ and $>30 \mu \mathrm{m}$ ), as well as the number of lysosomes in each cell (grouped by 1-5, 6-10, 11-15, 16-20, and $>20$ lysosomes/cell). $* * * * p<0.00001$ with ANOVA tests.

ApoE4 $4^{+/+}$miR-195: -0.144 versus $\left.0.231 ; p=0.024\right)$. The total amount of exploration time was comparable among all groups (data not shown).

Over-expressing miR-195 also reduced brain phosphoTau (pTau) levels in ApoE4 mouse brains (Fig. 4b; ApoE4 ${ }^{+/+}$scramble controls versus $A p o E 4^{+/+}$miR-195: 81.3 versus $39.1 \%$ of controls; $p=0.04$ ). Consistently, levels of synj1 mRNA and protein levels were reduced in ApoE4 $4^{+/+}$ mouse brains with miR-195 over-expression (Fig. S5A).
Trends of reduction but to lesser degrees in pTau, synj1 mRNA and protein levels were seen in $A p o E 3^{+/+}$mice with miR-195 over-expression. No significant changes were seen in endogenous $A \beta_{40}, A \beta_{42}$ or $A p o E$ levels with overexpression of miR-195 in $A p o E 4^{+/+}$or $A p o E 3^{+/+}$mouse brains (Fig. S5B and S5C). qPCR confirmed elevated miR195 levels after viral manipulations (Fig. S5D).

Similar experiments were performed in $\mathrm{ApoE}^{+/+}$or $A p o E 3^{+/+}$mouse models with $5 \times \mathrm{xAD}$ background with 
AD-related pathological, neuro-inflammatory, and behavioral phenotypes manifesting at 4-8 months of age $[48,49]$. Sex dimorphic responses were noted in these mouse models with male $\mathrm{ApoE} 4^{+/+} \mathrm{FAD}$ mice being most sensitive to miR-195 manipulations (Fig. 4c preference index: $\mathrm{ApoE}^{+/ /} \mathrm{FAD}$ scramble controls versus miR-195: $37.8 \%$ versus $63.1 \% ; p=0.01$; discrimination index: ApoE $4^{+/+}$scramble controls versus miR-195: -0.245 versus $0.262 ; p=0.016)$. $\mathrm{p}$-Tau reduction was also observed in ApoE $4^{+/+}$FAD mice with miR-195 over-expression (Figs. 4d and S5E). Similar changes were seen in total Tau levels in $\mathrm{ApoE}^{+/+} \mathrm{FAD}$ miR-195 over-expression mouse brains (data not shown). A dramatic reduction in brain oligomer $\mathrm{A} \beta_{42}$ measured by ELISA (Fig. 4e) and amyloid plaque burden determined by plaque numbers and plaque density (Fig. 4f) in $A p o E 4^{+/+}$and $A p o E 3^{+/+}$FAD mouse brains was found with miR-195 over-expression. However, no significant changes were seen in levels of soluble $A \beta_{40}$, $\mathrm{A} \beta_{42}$ (Fig. S5F), holo-APP or BACE-1 after miR-195 overexpression.

We also examined if miR-195 over-expression leads to changes in gene expression patterns and downstream pathways in EFAD mouse brains. Again, most differentially expressed genes (DEGs) are enriched in regulation of neuron, synapse, and immune functions (Table S3). Further GSEA studies [66] suggested top pathways perturbed by miR-195 over-expression are mitochondrial related pathways, consistent with studies in human brain dataset with top pathways enriched for genes negatively correlated with miR-195 involved in mitochondrial function (Table S2).

Together, our results suggest that elevating miR-195 levels in ApoE4 mouse models without and with $\mathrm{AD}$ background can rescue ApoE4- and AD-related cognitive deficits and pathological changes.

\section{Over-expression of miR-195 endo-lysosomal defects in iPSC-derived brain cells of ApoE4 AD subjects}

We next investigated if manipulating miR-195 levels could ameliorate AD-related pathologies using human induced pluripotent stem cells (hiPSCs)-derived neuron and astrocyte co-culture from $A p o E 4^{+/+} \mathrm{AD}$ subjects and $A p o E 3^{+/+}$normal aging subjects (TCW et al., bioRxiv; https://doi.org/10.1101/713362). At baseline, ApoE4 $4^{+/+}$ neurons (human iPSC or mouse) manifested enlarged lysosomes and increased numbers of lysosomes within each cell when compared to ApoE $3^{+/+}$counterparts (Fig. 5). The average size of lysosomes measured by area was $163.5 \mu^{2}$ in $\mathrm{ApoE}^{+/+}$versus $90.4 \mu^{2}$ in $\mathrm{ApoE}^{+/+}$ neurons (Fig. 5c, $p<0.00001$ ). There were $46.7 \%$ of ApoE $4^{+/+}$neurons with the diameter of lysosomes ranged from 10 to $20 \mu \mathrm{m}$, whereas $66.1 \%$ of $A p o E 3^{+/+}$neurons with the diameter ranged between 0 and $10 \mu \mathrm{m}$. There were $18.4 \% \mathrm{ApoE}^{+/+}$neurons with $>10$ lysosomes/cell, whereas only $1.6 \% \mathrm{ApoE}^{+/+}$neurons with $>10$ lysosomes/cell. Over-expression of miR-195 in ApoE4 $4^{+/+}$ neurons led to a significant reduction in lysosome size $\left(109.9 \mu \mathrm{m}^{2}, p<0.00001\right)$. The diameter of lysosomes and numbers of lysosomes per cell in $A p o E 4^{+/+}$neurons after miR-195 over-expression $(53.3 \%$ of neurons with the diameter ranged between 0 snd $10 \mu \mathrm{m} ; 1.7 \%$ with $>10$ lysosomes/cell) were also shifted toward the lysosomal phenotypes in $A p o E 3^{+/+}$neurons at baseline. In contrast, treatment with a miR-195 inhibitor exacerbated the lysosomal phenotypes of $A p o E 4^{+/+}$neurons (average size: $\left.205.4 \mu \mathrm{m}^{2}, p<0.00001\right)$ and increased numbers of lysosomes per cell $(40 \%$ of neurons with the diameter of lysosomes $>30 \mu \mathrm{m} ; 25 \%$ of cells with $>10$ lysosomes/ cell). No significant differences were seen in $A p o E 3^{+/+}$ neurons treated with miR-195 over-expression or inhibition when compared to the baseline. The pTau levels were reduced with over-expressing miR-195 and increased with miR-195 inhibition as demonstrated by fluorescent staining (Fig. S6A) and ELISA (Fig. S6B).

MiR-195 levels were also lower in cultured iPSC-derived astrocytes from $\mathrm{ApoE4^{+/+ }} \mathrm{AD}$ subjects when compared to those in $\mathrm{ApoE}^{+/+}$normal aging (NA) iPSC-derived astrocytes (Fig. S6C, $p=0.002$ ). The effects of miR-195 inhibitor on lysosomes can also be seen in $A p o E 4^{+/+}$astrocytes with a significant increase in average size of lysosomes (Fig. S6D, control versus miR-195 inhibitor treatment $36.5 \mu \mathrm{m}^{2}$ versus $67.7 \mu \mathrm{m}^{2}, p<0.00001$; Fig. S6E $8.3 \%$ of control astrocytes with the diameter of lysosomes $>30 \mu \mathrm{m}$ versus $17.6 \%$ of miR-195 inhibitor treated). However, no significant differences were seen in lysosomes of $\mathrm{ApoE}^{+/+}$ astrocytes with miR-195 treatment when compared to control, or between $A p o E 4^{+/+}$versus $A p o E 3^{+/+}$astrocytes at baseline.

Similar experiments were performed using mouse synj $1^{+/+}$and synj $1^{-/-}$neurons co-cultured with ApoE $4^{+/+}$ iPSC-derived astrocytes in the presence or absence of miR195 over-expression. We found that genetic knockout of synj1 manifested similar effects on lysosomal phenotypes as miR-195 over-expression. The average size of lysosomes was $101.5 \mu \mathrm{m}^{2}$ in synj $1^{+/+}$versus $77.0 \mu \mathrm{m}^{2}$ in synj1 $1^{-1-}$ neurons (Fig. S6F, $p<0.00001$ ). With miR-195 overexpression, the size of lysosomes was reduced to $75.8 \mu \mathrm{m}^{2}$ in synj $^{+/+}$neurons $(p<0.00001)$. However, overexpression of miR-195 in synj1 $1^{-1-}$ neurons did not exhibit any additive effects $\left(69.7 \mu^{2}\right)$, suggesting that miR-195 indeed acts through its target gene synjl to rescue ADrelated lysosomal defects.

Together, these results suggest that elevating miR-195 levels in human iPSC-derived ApoE4 $4^{+/+} \mathrm{AD}$ brain cells can rescue lysosomal defects, whereas inhibiting miR-195 can exacerbate these phenotypes. 


\section{Discussion}

$\mathrm{AD}$ is a complex, multifactorial neurodegenerative process, and accumulating evidence indicates the importance of miRNAs in AD pathogenesis. Our studies characterize the functional involvement of a miRNA, miR-195 in ApoE4associated pathology. Utilizing unbiased systems biology approaches as well as multiple computational prediction tools, we leverage existing human ROS-MAP miRNA datasets in combination with mouse micro-array studies and identify miR-195 as the top miRNA candidate in regulating the ApoE-synj1-PIP 2 pathways and that this regulation is differentially modulated by ApoE genotypes. More importantly, our data reveal a novel regulatory role of miR-195 in the ApoE4 genotype-associated cognitive and lysosomal defects that contribute to AD development.

Dysregulation in brain miRNAs has been described in human subjects and mouse models of $\mathrm{AD}$ with proposed involvement of $\mathrm{A} \beta$-dependent and $\mathrm{A} \beta$-independent pathways [36, 37, 73]. Our studies here demonstrate miR-195 reduction during $\mathrm{AD}$ development, which correlates with early disease progression but not with advanced stages of AD. ApoE4 genotype accelerates miR-195 reduction, which coincides with cognitive decline and increased tau pathology (Fig. 2). Previously, we reported patterns of changes in phosphoinositol (PI) metabolites correlated with disease conversion from normal aging to early AD [26]. Our results further indicate miR-195 changes are consistent with alternations in brain $\mathrm{PIP}_{2}$ levels (Fig. S2C). These findings highlight the potential of utilizing miR-195 levels as surrogate biomarkers to monitor brain $\mathrm{PIP}_{2}$ homeostasis and cognitive performance and detect early $\mathrm{AD}$ development and progression. Our studies of differentially expressed miRNAs between $A p o E 4^{+-}$and $A p o E 4^{-/-}$carriers in ROSMAP and mouse microarray datasets also identified another miRNA in common, miR-155. A prior report suggested a protective role of miR-155 in AD with increased brain miR-155 levels in aged AD transgenic mice (APP/PS1 or $\mathrm{Tau} \mathrm{Tg}$ ) and in $\mathrm{AD}$ human subjects compared to levels in controls [73]. On the other hand, our data show that miR155 is up-regulated in human $A p o E 4^{+/-}$carriers but downregulated in mouse ApoE4 condition (Fig. 1c). Nevertheless, the involvement of miR-155 in ApoE4 pathogenic function remains unclear with opposing changes in human and mouse datasets.

Interestingly, functional enrichment studies of human dataset (Table S2) and mouse transcriptomic dataset (Table S3) implicate the roles of miR-195 in regulating neuronal and synaptic function, neurogenesis, and differentiation. Consistently, our data indicate that restoring miR195 levels in vivo rescues ApoE4-associated cognitive deficits (Fig. 4a, c), ameliorates amyloid plaque burden (Fig. 4e, f) and pTau levels (Fig. 4b, d), and improves lysosomal defects in cultured human iPSC-derived brain cells (Fig. 5). While we did not see any reduction in endogenous mouse $\mathrm{A} \beta$ in ApoE KI mouse models (Fig. S5B) or soluble A $\beta$ levels in EFAD mice (Fig. S5F), we observed dramatic decreases in oligomer $A \beta$ levels and plaque burden with over-expression of miR-195 (Fig. 4e, f). In addition, we found a negative correlation between miR195 and BACE1 expression in human brain tissue $(r=$ $-0.516, p=0.004$; Fig. S2D), consistent with a previous report [67]. However, no changes in BACE-1 levels were seen with miR-195 over-expression (data not shown). Together, these data suggest that miR-195 most likely regulates $A \beta$ clearance instead of $A \beta$ generation, and restoration of lysosomal function may facilitate these processes, as indicated in our prior reports that synj1 reduction accelerates lysosomal clearance of $\mathrm{A} \beta$ [43].

Moreover, a functional role of miR-195 in regulating pTau levels is implicated (Fig. 4 and Fig. S6A and S6B), consistent with our recent findings that down-regulation of synj1 prevents mild TBI-induced tau hyper-phosphorylation [31]. It would be interesting to further investigate the molecular mechanisms by which miR-195 regulates tau hyper-phosphorylation. It was previously reported that the exosomal secretion of tau may play an important role in tau spread, which could be regulated by miRNAs [74]. We speculate that miR-195 might serve as the key in modulating tau pathology secondary to impaired clearance through the lysosomal pathway and/or accelerated spread through the exosomal secretory pathway.

The results suggest that differential regulation of miR-195 expression by $A p o E$ isoforms is mediated through binding to $A p o E$ receptors on neurons, and the $A p o E 4$ genotype loses the ability to regulate miR-195 levels (Fig. 3c). In addition, our data showing $A p o E^{-l-}$ with lower miR-195 expression like ApoE4 (Fig. 3a), further supports loss-of-function effects of ApoE4 on miR-195 expression leading to increased synjl mRNA and protein expression. Over-expression of miR-195 in synj $1^{-1-}$ neurons failing to exhibit any additive effects on lysosomal enlargement (Fig. S6F) further strengthens the concepts that miR-195 rescues AD-related phenotypes through its target gene, synj1. It should be noted that $\mathrm{ApoE4^{+/- }}$ carriers exhibit higher sensitivity to miR-195 manipulations than ApoE4 $4^{-1-}$ subjects (Figs. 4 and S5; Figs. 5 and S6), possibly due to much lower baseline miR-195 levels. Furthermore, differences in miR-195 levels between $\mathrm{ApoE}^{+/-}$and $A p o E 4^{-1-}$ conditions are not only seen in neurons but also in other brain cells such as astrocytes (Fig. S6C). The cell-type specific changes in miR-195 may contribute to different aspects of disease pathogenesis. We speculate that reduction in neuronal miR-195 levels may contribute to cognitive and synaptic dysfunction, while reduction in astrocytic miR-195 levels may play a role in defects in the secretory pathways leading to accelerated tau accumulation and spread. 
We also examined sex impact in miR-195 expression. In ROSMAP dataset, the differences in miR-195 levels between $A p o E 4^{+/-}$and $A p o E 4^{-/-}$carriers persist in female subjects (Fig. 1b), similarly to our previous report of sex-specific effects on brain $\mathrm{PIP}_{2}$ homeostasis [26]. Sex dimorphism is also noted in miR-195 expression with much lower levels in

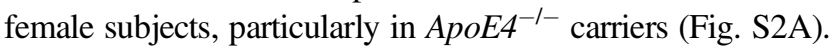
EFAD mice also exhibited sex dimorphic responses to miR195 manipulations with improved cognitive function and reduced oligomer $A \beta$ levels in male but not female EFAD mice (Fig. 4c, e). Age-related changes in miR-195 expression and synj 1 mRNA levels have also been noted in ApoE KI and EFAD mouse brains, with differential expression between $\mathrm{ApoE4}^{+/+}$and $\mathrm{ApoE4^{-/- }}$ mice more prominent at 12 months of age compared to a younger age, whereas differences in miR-195 in EFAD mice are already evident at 4 months of age (data not shown), suggesting that $A p o E$-genotype associated miR-195 changes might be exacerbated by aging and/ or manifestations of $\mathrm{AD}$ pathologies.

While AD manifests as a multi-faceted disease process, targeting a specific miRNA to restore dysregulated networks and pathways at multiple levels could provide a promising avenue for future drug development. Therapeutic strategies directed at ApoE4 have been and are actively explored in several preclinical and clinical studies such as immunotherapies, antisense oligonucleotide treatments, gene editing, modulators of $A p o E$ expression, as well as small molecules to enhance $A p o E$ lipidation, to correct its structures, to compete receptor binding, and to inhibit ApoE-A $\beta$ interaction [75, 76]. Our findings here suggest a novel therapeutic direction that modulates ApoE4 pathogenic function by a miRNA miR-195 through brain $\mathrm{PIP}_{2}$ lipid signaling pathways with multiple beneficial effects besides impact on $A \beta$ and tau pathology.

In summary, our studies provide a mechanistic link between ApoE4 genotype-specific changes in brain miR-195 expression with AD-related phenotypes including brain phospholipid dysregulation, cognitive deficits, lysosomal defects, and tau pathologies. These studies may uncover novel therapeutic strategies targeted at a specific miRNA miR-195.

\section{Data availability}

The human miRNA and RNA-seq datasets from ROSMAP studies are available via the AD Knowledge Portal (https://www.synapse.org/\#!Synapse:syn5856115 and https://www.synapse.org/\#!Synapse:syn3505720). The AD Knowledge Portal is a platform for accessing data, analyses, and tools generated by the Accelerating Medicines Partnership (AMP-AD) Target Discovery Program and other National Institute on Aging (NIA)-supported programs to enable open-science practices and accelerate translational learning. Data are available for general research use according to the following requirements for data access and data attribution (https://adknowledgeportal.synapse.org/ DataAccess/Instructions). The mouse microarray studies and RNA-seq datasets after miR-195 perturbation experiments generated in this paper have been deposited to the $\mathrm{AD}$ Knowledge Portal as well (https://www.synapse.org/\#!Syna pse:syn22123695).

Acknowledgements We thank Dr. Guojun Bu for generously providing us the RAP for our studies. This work was supported in whole or in part, by funding from NIH R01 (1R01AG048923) to DC, RF1 (RF1AG054014) and R56 (R56AG058655) to DC and BZ, Department of Veteran Affairs BLRD (I01BX003380) and RR\&D (I01RX002290) to DC, RRD (IO1RX002660) to GE, CSRD (I01CX000619) to MS, NIH K01 (AD062683) to J. TCW, NIH U01 (UO1AG046170) to BZ and VH, RF1 (RF1AG057440) to BZ and VH, UO1 (UO1AG052411) to $\mathrm{BZ}$ and $\mathrm{AG}$. Confocal microscopy studies and animal behavioral analysis were supported by James J. Peters VA Medical Center Research Core Facilities. Postmortem human brain tissue for validation studies were provided by NIH NeuroBioBank and CSF samples provided by JJP VAMC and ISMMS MCI research cohort.

\section{Compliance with ethical standards}

Conflict of interest The authors declare that they have no conflict of interest.

Publisher's note Springer Nature remains neutral with regard to jurisdictional claims in published maps and institutional affiliations.

Open Access This article is licensed under a Creative Commons Attribution 4.0 International License, which permits use, sharing, adaptation, distribution and reproduction in any medium or format, as long as you give appropriate credit to the original author(s) and the source, provide a link to the Creative Commons license, and indicate if changes were made. The images or other third party material in this article are included in the article's Creative Commons license, unless indicated otherwise in a credit line to the material. If material is not included in the article's Creative Commons license and your intended use is not permitted by statutory regulation or exceeds the permitted use, you will need to obtain permission directly from the copyright holder. To view a copy of this license, visit http://creativecommons. org/licenses/by/4.0/.

\section{References}

1. Mayeux R. Epidemiology of neurodegeneration. Annu Rev Neurosci. 2003;26:81-104.

2. Fagan AM, Watson M, Parsadanian M, Bales KR, Paul SM, Holtzman DM. Human and murine ApoE markedly alters A beta metabolism before and after plaque formation in a mouse model of Alzheimer's disease. Neurobiol Dis. 2002;9:305-18.

3. Jiang Y, Sun XC, Gui L, Tang WY, Zhen LP, Gu YJ, et al. Lack of association between apolipoprotein E promoters in epsilon4 carriers and worsening on computed tomography in early stage of traumatic brain injury. Acta Neurochir Suppl. 2008;105:233-6.

4. Jordan J, Galindo MF, Miller RJ, Reardon CA, Getz GS, LaDu MJ. Isoform-specific effect of apolipoprotein $E$ on cell survival and beta-amyloid-induced toxicity in rat hippocampal pyramidal neuronal cultures. J Neurosci: Off J Soc Neurosci. 1998;18:195-204. 
5. Ma J, Yee A, Brewer HB Jr, Das S, Potter H. Amyloid-associated proteins alpha 1-antichymotrypsin and apolipoprotein $\mathrm{E}$ promote assembly of Alzheimer beta-protein into filaments. Nature. 1994;372:92-4.

6. Sharman MJ, Morici M, Hone E, Berger T, Taddei K, Martins IJ, et al. APOE genotype results in differential effects on the peripheral clearance of amyloid-beta42 in APOE knock-in and knock-out mice. J Alzheimer's Dis: JAD. 2010;21:403-9.

7. Yang DS, Smith JD, Zhou Z, Gandy SE, Martins RN. Characterization of the binding of amyloid-beta peptide to cell culturederived native apolipoprotein E2, E3, and E4 isoforms and to isoforms from human plasma. J neurochemistry. 1997;68:721-5.

8. Oyama F, Shimada H, Oyama R, Ihara Y. Apolipoprotein E genotype, Alzheimer's pathologies and related gene expression in the aged population. Brain Res Mol Brain Res. 1995;29:92-8.

9. Shi Y, Yamada K, Liddelow SA, Smith ST, Zhao L, Luo W, et al. ApoE4 markedly exacerbates tau-mediated neurodegeneration in a mouse model of tauopathy. Nature. 2017;549:523-7.

10. Tiraboschi P, Hansen LA, Masliah E, Alford M, Thal LJ, CoreyBloom J. Impact of APOE genotype on neuropathologic and neurochemical markers of Alzheimer disease. Neurology. 2004;62:1977-83.

11. Wang Y, Mandelkow E. Tau in physiology and pathology. Nat Rev Neurosci. 2016;17:5-21.

12. Love S, Siew LK, Dawbarn D, Wilcock GK, Ben-Shlomo Y, Allen SJ. Premorbid effects of APOE on synaptic proteins in human temporal neocortex. Neurobiol Aging. 2006;27:797-803.

13. Nathan BP, Bellosta S, Sanan DA, Weisgraber KH, Mahley RW, Pitas RE. Differential effects of apolipoproteins E3 and E4 on neuronal growth in vitro. Science. 1994;264:850-2.

14. Teter B, Xu PT, Gilbert JR, Roses AD, Galasko D, Cole GM. Human apolipoprotein $\mathrm{E}$ isoform-specific differences in neuronal sprouting in organotypic hippocampal culture. J Neurochem. 1999;73:2613-6.

15. Trommer BL, Shah C, Yun SH, Gamkrelidze G, Pasternak ES, Ye GL, et al. ApoE isoform affects LTP in human targeted replacement mice. Neuroreport. 2004;15:2655-8.

16. Keren-Shaul H, Spinrad A, Weiner A, Matcovitch-Natan O, DvirSzternfeld R, Ulland TK, et al. A unique microglia type associated with restricting development of Alzheimer's disease. Cell. 2017;169:1276-90 e1217.

17. Yeh FL, Wang Y, Tom I, Gonzalez LC, Sheng M. TREM2 binds to apolipoproteins, including APOE and CLU/APOJ, and thereby facilitates uptake of amyloid-beta by microglia. Neuron. 2016;91:328-40.

18. Zhu Y, Nwabuisi-Heath E, Dumanis SB, Tai LM, Yu C, Rebeck $\mathrm{GW}$, et al. APOE genotype alters glial activation and loss of synaptic markers in mice. Glia. 2012;60:559-69.

19. Huang Y, Mahley RW, Apolipoprotein E. structure and function in lipid metabolism, neurobiology, and Alzheimer's diseases. Neurobiol Dis. 2014;72(Pt A):3-12.

20. Pettegrew JW, Panchalingam K, Hamilton RL, McClure RJ. Brain membrane phospholipid alterations in Alzheimer's disease. Neurochem Res. 2001;26:771-82.

21. Mandal PK, McClure RJ, Pettegrew JW. Interactions of Abeta(140) with glycerophosphocholine and intact erythrocyte membranes: fluorescence and circular dichroism studies. Neurochem Res. 2004;29:2273-9.

22. Kanfer JN, Pettegrew JW, Moossy J, McCartney DG. Alterations of selected enzymes of phospholipid metabolism in Alzheimer's disease brain tissue as compared to non-Alzheimer's demented controls. Neurochem Res. 1993;18:331-4.

23. Kanfer JN, Singh IN, Pettegrew JW, McCartney DG, Sorrentino G. Phospholipid metabolism in Alzheimer's disease and in a human cholinergic cell. J Lipid Mediat Cell Signal. 1996;14:361-3.
24. Chan RB, Oliveira TG, Cortes EP, Honig LS, Duff KE, Small SA, et al. Comparative lipidomic analysis of mouse and human brain with Alzheimer disease. J Biol Chem. 2012;287:2678-88.

25. Klunk WE, Panchalingam K, McClure RJ, Stanley JA, Pettegrew JW. Metabolic alterations in postmortem Alzheimer's disease brain are exaggerated by Apo-E4. Neurobiol aging. 1998;19:511-5.

26. Zhu L, Zhong M, Elder GA, Sano M, Holtzman DM, Gandy S, et al. Phospholipid dysregulation contributes to ApoE4-associated cognitive deficits in Alzheimer's disease pathogenesis. Proc Natl Acad Sci USA. 2015;112:11965-70.

27. Berman DE, Dall'Armi C, Voronov SV, McIntire LB, Zhang H, Moore AZ, et al. Oligomeric amyloid-beta peptide disrupts phosphatidylinositol-4,5-bisphosphate metabolism. Nat Neurosci. 2008;11:547-54.

28. Voronov SV, Frere SG, Giovedi S, Pollina EA, Borel C, Zhang H, et al. Synaptojanin 1-linked phosphoinositide dyshomeostasis and cognitive deficits in mouse models of Down's syndrome. Proc Natl Acad Sci USA. 2008;105:9415-20.

29. McIntire LB, Berman DE, Myaeng J, Staniszewski A, Arancio O, Di Paolo G, et al. Reduction of synaptojanin 1 ameliorates synaptic and behavioral impairments in a mouse model of Alzheimer's disease. J Neurosci: Off J Soc Neurosci. 2012;32:15271-6.

30. Zhu L, Zhong M, Zhao J, Rhee H, Caesar I, Knight E, et al. Reduction of synaptojanin 1 accelerates Abeta clearance and attenuates cognitive deterioration in an Alzheimer mouse model. $\mathrm{J}$ Biol Chem. 2013;288:32050-63.

31. Cao J, Gaamouch FE, Meabon JS, Meeker KD, Zhu L, Zhong $\mathrm{MB}$, et al. ApoE4-associated phospholipid dysregulation contributes to development of Tau hyper-phosphorylation after traumatic brain injury. Sci Rep. 2017;7:11372.

32. Miranda AM, Herman M, Cheng R, Nahmani E, Barrett G, Micevska E, et al. Excess Synaptojanin 1 contributes to place cell dysfunction and memory deficits in the aging hippocampus in three types of Alzheimer's disease. Cell Rep. 2018;23:2967-75.

33. Cossec JC, Lavaur J, Berman DE, Rivals I, Hoischen A, Stora S, et al. Trisomy for synaptojanin1 in Down syndrome is functionally linked to the enlargement of early endosomes. Hum Mol Genet. 2012;21:3156-72.

34. Fabian MR, Sonenberg N, Filipowicz W. Regulation of mRNA translation and stability by microRNAs. Annu Rev Biochem. 2010;79:351-79.

35. Wang M, Qin L, Tang B. MicroRNAs in Alzheimer's disease. Front Genet. 2019;10:153.

36. Goodall EF, Heath PR, Bandmann O, Kirby J, Shaw PJ. Neuronal dark matter: the emerging role of microRNAs in neurodegeneration. Front Cell Neurosci. 2013;7:178.

37. Lukiw WJ. Micro-RNA speciation in fetal, adult and Alzheimer's disease hippocampus. Neuroreport. 2007;18:297-300.

38. Bennett DA, Schneider JA, Buchman AS, Mendes de Leon C, Bienias JL, Wilson RS. The rush memory and aging project: study design and baseline characteristics of the study cohort. Neuroepidemiology. 2005;25:163-75.

39. Bennett A, Schneider J, Arvanitakis Z, Wilson R. Overview and findings from the religious orders study. Curr Alzheimer Res. 2012;9:628-45.

40. Wong N, Wang X. miRDB: an online resource for microRNA target prediction and functional annotations. Nucleic acids Res. 2015;43:D146-52.

41. Wang $X$. Improving microRNA target prediction by modeling with unambiguously identified microRNA-target pairs from CLIPLigation studies. Bioinformatics. 2016;32:1316-22.

42. Smyth GK. Linear models and empirical bayes methods for assessing differential expression in microarray experiments. Stat Appl Genet Mol Biol. 2004;3:3.

43. Zhu L, Zhong M, Zhao J, Rhee H, Caesar I, Knight EM, et al. Reduction of synaptojanin 1 accelerates Abeta clearance and 
attenuates cognitive deterioration in an Alzheimer mouse model. J Biol Chem. 2013;288:32050-63.

44. Ru Y, Kechris KJ, Tabakoff B, Hoffman P, Radcliffe RA, Bowler $\mathrm{R}$, et al. The multiMiR $\mathrm{R}$ package and database: integration of microRNA-target interactions along with their disease and drug associations. Nucleic acids Res. 2014;42:e133.

45. Grootendorst J, Bour A, Vogel E, Kelche C, Sullivan PM, Dodart JC, et al. Human apoE targeted replacement mouse lines: h-apoE4 and h-apoE3 mice differ on spatial memory performance and avoidance behavior. Behav Brain Res. 2005;159:1-14.

46. Rodriguez GA, Burns MP, Weeber EJ, Rebeck GW. Young APOE4 targeted replacement mice exhibit poor spatial learning and memory, with reduced dendritic spine density in the medial entorhinal cortex. Learn Mem. 2013;20:256-66.

47. Wang C, Wilson WA, Moore SD, Mace BE, Maeda N, Schmechel $\mathrm{DE}$, et al. Human apoE4-targeted replacement mice display synaptic deficits in the absence of neuropathology. Neurobiol Dis. 2005; 18:390-8.

48. Balu D, Karstens AJ, Loukenas E, Maldonado Weng J, York JM, Valencia-Olvera AC, et al. The role of APOE in transgenic mouse models of AD. Neurosci Lett. 2019;707:134285.

49. Tai LM, Balu D, Avila-Munoz E, Abdullah L, Thomas R, Collins N, et al. EFAD transgenic mice as a human APOE relevant preclinical model of Alzheimer's disease. J Lipid Res. 2017;58:1733-55.

50. Zhong L, Xie YZ, Cao TT, Wang Z, Wang T, Li X, et al. A rapid and cost-effective method for genotyping apolipoprotein $\mathrm{E}$ gene polymorphism. Mol Neurodegener. 2016;11:2.

51. Nasuhoglu C, Feng S, Mao J, Yamamoto M, Yin HL, Earnest S, et al. Nonradioactive analysis of phosphatidylinositides and other anionic phospholipids by anion-exchange high-performance liquid chromatography with suppressed conductivity detection. Anal Biochem. 2002;301:243-54.

52. Berg I, Nilsson KP, Thor S, Hammarstrom P. Efficient imaging of amyloid deposits in Drosophila models of human amyloidoses. Nat Protoc. 2010;5:935-44.

53. Bowles KR, Tcw J, Qian L, Jadow BM, Goate AM. Reduced variability of neural progenitor cells and improved purity of neuronal cultures using magnetic activated cell sorting. PloS One. 2019;14:e0213374.

54. Tcw J, Wang M, Pimenova AA, Bowles KR, Hartley BJ, Lacin E, et al. An efficient platform for astrocyte differentiation from human induced pluripotent stem cells. Stem Cell Rep. 2017;9:600-14.

55. Bardy C, van den Hurk M, Eames T, Marchand C, Hernandez RV, Kellogg M, et al. Neuronal medium that supports basic synaptic functions and activity of human neurons in vitro. Proc Natl Acad Sci USA. 2015;112:E2725-34.

56. Paquet D, Kwart D, Chen A, Sproul A, Jacob S, Teo S, et al. Efficient introduction of specific homozygous and heterozygous mutations using CRISPR/Cas9. Nature. 2016;533:125-9.

57. Shaltouki A, Peng J, Liu Q, Rao MS, Zeng X. Efficient generation of astrocytes from human pluripotent stem cells in defined conditions. Stem Cells. 2013;31:941-52.

58. Labonte B, Engmann O, Purushothaman I, Menard C, Wang J, Tan C, et al. Sex-specific transcriptional signatures in human depression. Nat Med. 2017;23:1102-11.

59. Passini MA, Dodge JC, Bu J, Yang W, Zhao Q, Sondhi D, et al. Intracranial delivery of CLN2 reduces brain pathology in a mouse model of classical late infantile neuronal ceroid lipofuscinosis. J Neurosci: Off J Soc Neurosci. 2006;26:1334-42.

60. Elder GA, Dorr NP, De Gasperi R, Gama Sosa MA, Shaughness $\mathrm{MC}$, Maudlin-Jeronimo E, et al. Blast exposure induces post- traumatic stress disorder-related traits in a rat model of mild traumatic brain injury. J Neurotrauma. 2012;29:2564-75.

61. Howlett DR, Richardson JC, Austin A, Parsons AA, Bate ST, Davies DC, et al. Cognitive correlates of Abeta deposition in male and female mice bearing amyloid precursor protein and presenilin1 mutant transgenes. Brain Res. 2004;1017:130-6.

62. Lane RF, Raines SM, Steele JW, Ehrlich ME, Lah JA, Small SA, et al. Diabetes-associated SorCS1 regulates Alzheimer's amyloidbeta metabolism: evidence for involvement of SorL1 and the retromer complex. J Neurosci: Off $J$ Soc Neurosci. 2010;30:13110-5.

63. Kawarabayashi T, Younkin LH, Saido TC, Shoji M, Ashe KH, Younkin SG. Age-dependent changes in brain, CSF, and plasma amyloid (beta) protein in the $\mathrm{Tg} 2576$ transgenic mouse model of Alzheimer's disease. J Neurosci: Off J Soc Neurosci. $2001 ; 21: 372-81$

64. Liao Y, Smyth GK, Shi W. FeatureCounts: an efficient general purpose program for assigning sequence reads to genomic features. Bioinformatics. 2014;30:923-30.

65. Robinson MD, Oshlack A. A scaling normalization method for differential expression analysis of RNA-seq data. Genome Biol. 2010;11:R25.

66. Subramanian A, Tamayo P, Mootha VK, Mukherjee S, Ebert BL, Gillette MA, et al. Gene set enrichment analysis: a knowledgebased approach for interpreting genome-wide expression profiles. Proc Natl Acad Sci USA. 2005;102:15545-50.

67. Zhu HC, Wang LM, Wang M, Song B, Tan S, Teng JF, et al. MicroRNA-195 downregulates Alzheimer's disease amyloid-beta production by targeting BACE1. Brain Res Bull. 2012;88:596-601.

68. Monteiro-Cardoso VF, Oliveira MM, Melo T, Domingues MR, Moreira PI, Ferreiro E, et al. Cardiolipin profile changes are associated to the early synaptic mitochondrial dysfunction in Alzheimer's disease. J Alzheimer's Dis: JAD. 2015;43:1375-92.

69. Chicco AJ, Sparagna GC. Role of cardiolipin alterations in mitochondrial dysfunction and disease. Am J Physiol Cell Physiol. 2007;292:C33-44.

70. LaDu MJ, Shah JA, Reardon CA, Getz GS, Bu G, Hu J, et al. Apolipoprotein $\mathrm{E}$ and apolipoprotein $\mathrm{E}$ receptors modulate A betainduced glial neuroinflammatory responses. Neurochemistry Int. 2001;39:427-34.

71. Qiu Z, Hyman BT, Rebeck GW. Apolipoprotein E receptors mediate neurite outgrowth through activation of p44/42 mitogenactivated protein kinase in primary neurons. J Biol Chem. 2004;279:34948-56.

72. Antunes M, Biala G. The novel object recognition memory: neurobiology, test procedure, and its modifications. Cogn Process. 2012;13:93-110.

73. Sierksma A, Lu A, Salta E, Vanden Eynden E, Callaerts-Vegh Z, D'Hooge R, et al. Deregulation of neuronal miRNAs induced by amyloid-beta or TAU pathology. Mol Neurodegener. 2018;13:54.

74. Asai H, Ikezu S, Tsunoda S, Medalla M, Luebke J, Haydar T, et al. Depletion of microglia and inhibition of exosome synthesis halt tau propagation. Nat Neurosci. 2015;18:1584-93.

75. Cao J, Hou J, Ping J, Cai D. Advances in developing novel therapeutic strategies for Alzheimer's disease. Mol Neurodegener. 2018;13:64.

76. Williams T, Borchelt DR, Chakrabarty P. Therapeutic approaches targeting Apolipoprotein E function in Alzheimer's disease. Mol Neurodegener. 2020;15:8. 